\title{
Distributed Opportunistic Sensing in Mobile Phone Sensor Networks
}

\author{
Viet-Duc Le \\ Pervasive Systems Group \\ University of Twente \\ Enschede, The Netherlands \\ v.d.le@utwente.nl
}

\begin{abstract}
The advantages of smartphones such as integrated sensors, programmability, scalability and cloud servers have enabled low-cost and efficient public safety applications. However, designing such applications has to face daunting challenges, for instance, short battery life, low computing capability and lacking memory. To this end, lighter, faster, more efficient and scalable distributed data processing algorithms are necessary. In particular, new mechanisms are necessary for adaptive and opportunistic sensing within a cluster of smartphones. Furthermore, a distributed data mining algorithm is in need of proposition to run on each smartphone for information retrieval. The retrieved information also needs a new algorithm to be fused and even enhanced by more powerful devices such as sensor nodes in the infrastructure to obtain higher accuracy and reliability. For experimental validation, the new algorithms will be implemented on smartphones to collect and detect abnormalities from measured environmental elements such as sounds, pressure, temperature, light, etc., under various public safety scenarios including ambient noise. Currently, I am working from the scratch on a new idea that using a few preferable sensors to trigger others' measurement for adaptive and opportunistic sensing. This saves considerable battery power and lessens interference with normal phone usage.
\end{abstract}

Keywords-addaptive sensing; opportunistic sensing; distributed data mining; anomaly detection; smartphones.

\section{INTRODUCTION}

The significantly developing smartphones has invited researchers to develop opportunistic mobile sensor network applications due to a number of advantages[1][2]. First, the increasing amount of onboard sensors on smartphones, which were initially embedded to comfort users such as a light sensor to switch off screen during talking, enables building low-cost sensing applications. Second, the powerful processing capability such as the quad-core chip in the smartphone Galaxy Note II allows implementing heavier computing algorithms compared to conventional cheap wireless sensor modules. Third, Google, Apple and Samung stores facilitate deploying sensors in large scale via delivering apps to an enormous number of people. Fourth, the powerful computation and gigantic storage of cloud servers, which are strongly linked to smartphones, support a deeper level of learning such as users' behavior based on data mining from huge database collected through smartphones.

Due to the special characteristics of smartphones, design- ing sensing schemes in dynamic systems such as Funiak et al. [3] needs to be compromised with energy consumption, computation, memory, user usage and information. Energy consumption should be kept to a minimum due to the strictly limited battery life. Other resources such as memory and processing time should also be considered. In addition, the applications have to be transparent or keep least jeopardizing the normal phone usage. Moreover, security schemes must be developed to prevent leaking personal information while using sensing applications.

Since we consider opportunistic networks that have sparse density and intermittent connectivity, collecting and then processing data at a central server results in high resource consumption and outdated trained model. Therefore, my research focuses on developing new algorithms for distributed data mining for opportunistic sensing systems using integrated sensors in smartphones. External sensors, for instance, gas and dust sensors, are probably needed in future. This research will make conventional public safety applications, which require high density of expensive sensors, practical using low-cost sensors integrated in smartphones.

In the rest of this extended abstract, Section II formulates research problems. My abstract approaches are discussed in Section III. Section IV briefly presents my ongoing work. Finally, conclusions in Section V will end this paper.

\section{Problem Formulation}

My research consists of four main problems: adaptive sampling, opportunistic sensing, distributed data mining and advanced event detection.

- Adaptive sampling: Given a set of fusion sensors of a group of smartphones, the problem is how to switch on and off suitable sensors at moments when some abnormal might happen to reduce power consumption.

- Opportunistic sensing: Given fusion data from a set of smarphones, the problem is how to share such data among smartphones to enhance the reliability by increasing variety of sensor types since the measurement accuracy and the set of sensors vary with types of smartphones.

- Distributed data mining: Given fusion data collected from neighboring smartphones, the problem is how to do distributed data mining on suitable smartphones 


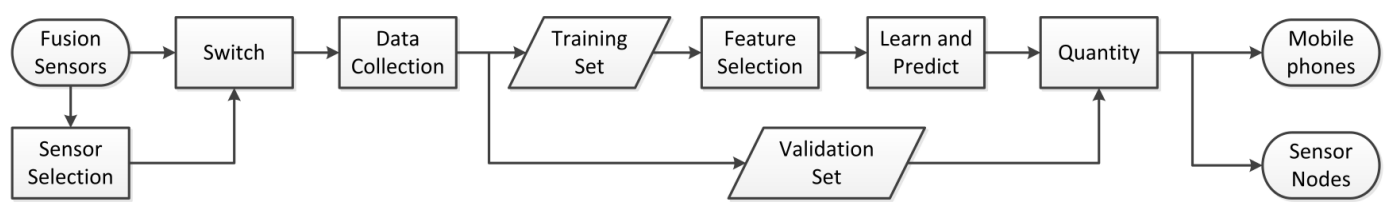

Figure 1. Distributed Opportunistic Sensing for Mobile Phone Sensor Networks

while still be almost transparent to users, specially in terms of power consumption and phone usage.

- Advanced event detection: Given fusion data and preliminary retrieved information from clusters of smartphones, the problem is how to integrate them with measurements from more powerful devices such as sensor modules to better recognize contexts.

\section{APPROACHES}

In this section, I interpret briefly how my conceptual approach that is depicted in Figure 1 would solve four problems defined in Section II.

\section{A. Adaptive Sensing}

Sensor Selection selects a subset of sensors from available ones to monitor environmental context. If there is an outline detected from measurements, a trigger will be generated to switch on more types of sensors in a short period. This approach saves considerable power consumption because most sensors are frequently off. Note that adapting sampling rates and sets of sensors has been used to save energy in sensor networks are not effective when available sensors and environmental contexts are highly dynamic and unpredictable.

\section{B. Opportunistic Sensing}

Data Collection collects sensor data from neighboring smartphones in an opportunistic manner. For example, a smartphone that does not have ambient temperature data can use measurements of adjacent ones to enrich information. The gathered data will be split into two separated sets for training and validation. In fact, adaptive sensing and opportunistic sensing processes strongly link together. These processes should be executed simultaneously to save the number of transmissions and power consumption.

\section{Distributed Data Mining}

Distributed data mining algorithm runs on the Learn and Predict block to estimate the local environment situations. Built models for the data mining can be improved and validated via the Quantity block, which includes metrics for evaluation. Training is split into multi-tasks that can be assigned for smartphones and local infrastructures, which compose an opportunistic network.

\section{Advanced Event Detection}

The consensus data and preliminarily obtained contextawareness from the Learn and Predict block are disseminated to powerful sensors in infrastructures and central servers to be improved in terms of reliability and accuracy based on advanced machine learning algorithms.

\section{ONGOING WORK}

Started few months ago, I am finishing the adaptive sensing problem. I proposed a new anomaly detection algorithm based on historical features of data measured by microphones, light sensors and accelerometers, which are empirically chosen because they quickly respond to most events, to trigger other sensors. To evaluate the algorithm, sound effects collected from the Internet are mixed with normally environmental audio recorded by myself with various scenarios. To create abnormal events, these mixed sounds are played via speakers in a room, which also has some fire and vibration generators. If the results are as expected, I will set up a more realistic test-bed to investigate further my algorithm.

\section{Conclusions}

This extended abstract briefly introduces my research topic, distributed data processing in opportunistic mobile phone networks. There are four main research problems related to adaptive and opportunistic sensing, opportunistic and distributed data mining, and advanced anomaly detection. The results of my research are deliberately applied for, but not limited to, public safety applications such as fire detection and disaster management for faster and more accurately responses.

\section{ACKNOWLEDGMENT}

This work is supported by the SenSafety project in the Dutch Commit program. I would like to thank Hans Scholten and Paul Havinga from the Pervasive Systems Group, University of Twente, for their supervision.

\section{REFERENCES}

[1] N. D. Lane, E. Miluzzo, H. Lu, D. Peebles, T. Choudhury, and A. T. Campbell, "A survey of mobile phone sensing," Comm. Mag., vol. 48, pp. 140-150, 2010.

[2] V.-D. Le, H. Scholten, and P. Havinga, "Unified routing for data dissemination in smart city networks," in Proc. of the 3rd International Conference on the Internet of Things (IoT2012), 2012.

[3] S. Funiak, C. Guestrin, M. Paskin, and R. Sukthankar, "Distributed inference in dynamical systems," in Advances in Neural Information Processing Systems 19, B. Scholkopf, J. Platt, and T. Hoffman, Eds. Cambridge, MA: MIT Press, December 2006, pp. 433-440. 